\title{
Cultura, políticas públicas Y BIENES COMUNES: HACIA UNAS POLÍTICAS DE LO CULTURAL
}

\author{
Nicolás Barbieri \\ Instituto de Gobierno y Políticas Públicas, \\ Universitat Autònoma de Barcelona
}

RESUMEN: Las políticas culturales se han institucionalizado sobre la idea de la cultura como sustantivo, como objeto, incluso como esencia de un grupo o colectivo. Si bien el modelo de democratización de la cultura continúa como núcleo de las políticas culturales, se vuelve evidente la caducidad de buena parte de los principios de una política centrada en el acceso a la oferta cultural. Los problemas a los que las políticas públicas se enfrentan resultan cada vez más complejos, inciertos y con más riesgos incorporados. Suponen un nuevo reto para la legitimidad de las instituciones culturales en su rol de intermediación tradicional. En este contexto, los bienes comunes (commons) emergen no sólo como concepto analítico sino como herramientas de acción política, social y legal. Los bienes comunes no son simplemente objetos o espacios, sino complejos sistemas de gestión de recursos con comunidades y normas que permiten su sostenibilidad. Este artículo plantea como hipótesis la posibilidad de que las políticas culturales reconozcan la cultura no sólo como sustantivo sino también como adjetivo (lo cultural). ¿Qué implica concebir las políticas culturales como políticas de los bienes comunes? ¿Cuáles son las diferencias principales en lo que se refiere al modelo de políticas de acceso a la cultura? ¿Puede un cambio de modelo derivar en la desresponsabilización de las políticas públicas en el ámbito de la cultura?

Palabras claVe: políticas culturales, bienes comunes, acceso, políticas públicas.

RESUM: Les polítiques culturals s'han institucionalitzat sobre la idea de la cultura com a substantiu, com a objecte, fins i tot com a essència d'un grup o 
col-lectiu. Tot i que el model de democratització de la cultura continua com a nucli de les polítiques culturals, es torna evident la caducitat de bona part dels principis d'una política centrada a l'accés a l'oferta cultural. Els problemes a què les polítiques públiques s'enfronten resulten cada vegada més complexos, incerts i amb més riscos incorporats. Suposen un nou repte per a la legitimitat de les institucions culturals en el seu rol d'intermediació tradicional. En aquest context, els béns comuns (commons) emergeixen no sols com a concepte analític sinó com a eines d'acció política, social i legal. Els béns comuns no són simplement objectes o espais sinó complexos sistemes de gestió de recursos amb comunitats i normes que permeten la seua sostenibilitat. Aquest article planteja com a hipòtesi la possibilitat que les polítiques culturals reconeguen la cultura no sols com a substantiu sinó també com a adjectiu (allò cultural). Què implica concebre les polítiques culturals com a polítiques dels béns comuns? Quines són les diferències principals en relació al model de polítiques d'accés a la cultura? Pot un canvi de model derivar en la desresponsabilització de les polítiques públiques en l'àmbit de la cultura?

PARAULES CLAU: polítiques culturals, béns comuns, accés, polítiques públiques.

ABSTRACT: Cultural policies have been institutionalised around the idea of culture as a noun, as an object, and even as the essence of a group or collective. While the model of the democratisation of culture remains at the heart of cultural policies, the temporary nature of many of the principles of a policy focused on access to cultural activities is becoming apparent. The problems faced by public policies are increasingly complex, uncertain and involve more and more risks. They pose a new challenge to the legitimacy of cultural institutions in their traditional role of intermediation. In this context, the commons are not merely an analytical concept, but are also tools for political, social and legal action. They are not just objects or spaces, but also complex systems for resource management with communities and rules that allow their sustainability. This article considers the hypothesis of the possibility of cultural policies recognising culture not only as a noun but as an adjective (the cultural). What does conceiving of cultural policies in terms of policies of the commons entail? What are the main differences in the model of policies for access to culture? Can a 
change of model lead to the removal of responsibility from public policies in the field of culture?

KEYWORDs: cultural policies, commons, access, public policies.

\section{Políticas públicas, cultura y ciudad}

$\mathrm{H}^{a}$ a sido ampliamente estudiado el proceso por el cual el principio de democratización de la cultura guía y legitima la mayoría de políticas culturales desarrolladas en Europa y en el mundo anglosajón a partir de la década de 1960. Este paradigma, así como el contrato que comporta entre agentes culturales públicos y privados, es de carácter eminentemente estatal-nacional. Es decir, los ministerios de cultura o los consejos de las artes estatales, según el modelo continental o anglosajón, son desde entonces los agentes referentes en el diseño e implementación de las políticas culturales. Estamos, en definitiva, frente al período de institucionalización de la política cultural como una política pública. No cabe duda de que con debilidades, y muy importantes, si se la compara con otras políticas públicas, incluso en el ámbito social. Pero a pesar de estas dificultades, la política cultural se establece como objeto de la intervención estatal, como instrumento de intervención gubernamental.

Con el proceso de institucionalización de las políticas culturales se consolida en esos años el modelo de políticas de acceso a la cultura. La intervención gubernamental se centra en la promoción de la oferta cultural entendida como de calidad y en la protección del patrimonio. Es en este período donde podemos identificar uno de los dilemas clave en la evolución de las políticas culturales: la necesidad de congeniar el fomento de la excelencia y la democratización. Prueba de ello es el lema que inspiraba el inicio del Arts Council of Great Britain en 1946: «lo mejor para la mayoría». En el intento de popularizar las llamadas artes eruditas, se impulsan infraestructuras culturales: de los museos a las bibliotecas, de los teatros a la monumentalización del espacio público. Son las décadas de los grandes equipamientos culturales, así como de la producción de oferta cultural directa por parte del Estado. Tam- 
bién es la etapa de consolidación institucional de la relación entre política cultural e identidad nacional.

Por último, este período es también el de consolidación de una dicotomía clave para entender la evolución de las políticas culturales. Se trata de la dicotomía Estado-mercado, que no sólo excluye el desarrollo de modelos alternativos sino que implica la asociación entre lo estatal y lo público, por un lado, y lo mercantil y lo privado, por otro.

Al modelo de democratización se añade pronto un tipo de políticas públicas que incorpora la promoción de espacios de participación y expresión sociocultural. Un modelo de políticas calificado como de democracia cultural (Urfalino, 1996). Pero la participación masiva, tal y como se entiende en el modelo de la democracia cultural, no se produce (Wu, 2007). Y son los gobiernos quienes se reafirman en el rol de productores culturales: el ámbito institucional asume el papel de lo público.

En este contexto se consolida la idea de que los gobiernos locales están en iguales o mejores condiciones para emprender las tareas de promoción de la cultura. Los objetivos e instrumentos tradicionales de la política cultural ceden terreno a nuevas lógicas de intervención que tienen origen en el ámbito local: adopción de una perspectiva sistémica de la cultura, expansión de la agenda de problemas abordados o intento de establecer un gobierno multinivel y el modelo de gobernanza de la cultura (Cherbo y Wyszomirski, 2000; Rodríguez Morató, 2005; Bonet y Negrier, 2008; Rius, 2012). En definitiva, la consolidación de gobiernos regionales y locales, así como el desarrollo del sector privado y del tercer sector, comporta también las demandas de cesión de parte de la agencia de las políticas (Barbieri et al., 2012).

Pero este contexto es también el del cuestionamiento de la idea de cultura como derecho fundamental que necesita ser garantizado por el servicio público. Con las críticas al papel del Estado como garante del bienestar se cuestiona también la autonomía del arte y la cultura: se presentan como un producto más que compite por el tiempo, interés y dinero del consumidor, y que por lo tanto deben demostrar su utilidad social y económica.

En definitiva, si hablamos de cultura y ciudad, si con la generalización de lo urbano las ciudades van perdiendo su significación autónoma, su capacidad 
de ser promesas de integración y liberación (Subirats y Blanco, 2009), la cultura pierde su estatus de vía para conseguir la libertad, la identidad nacional y la universalidad de la ciudadanía.

¿Cuáles han sido las respuestas más evidentes por parte de los poderes públicos en el ámbito de la cultura frente a este conjunto de fenómenos? Los procesos descritos tienen repercusiones significativas sobre la legitimidad del modelo de políticas de acceso a la cultura. Si bien el modelo de democratización de la cultura continúa actualmente como núcleo de la intervención gubernamental, se vuelve evidente la caducidad de buena parte de los principios de una política centrada en el acceso a la oferta cultural. Aparecerán entonces nuevas lógicas de justificación de la intervención gubernamental en el ámbito de la cultura y, en particular, en el espacio urbano.

\section{Políticas de la cultura, políticas de los objetos}

Frente a los cuestionamientos apuntados, los gobiernos optan por dinámicas de relegitimación de las políticas culturales construidas sobre respuestas que entendemos como reactivas, defensivas. Tanto en el ámbito económico como en el social. Por un lado, bajo la presión del creciente nivel de competencia entre ciudades basado en criterios de desarrollo económico, buena parte de los gobiernos resignifican el territorio como palanca de crecimiento económico. La cultura y las políticas culturales están en el centro de este proceso.

En este sentido, una de las miradas más adoptadas y a la vez más discutidas en las agendas políticas es la teoría de las clases y las ciudades creativas (Florida, 2002; Knudsen et al., 2007). Se trata de un modelo ampliamente contestado (Hall, 2004; Glaeser, 2005; Pratt, 2008), que incorpora la mercantilización progresiva de los recursos culturales y de la misma idea de convivencia (en forma de branding urbano) como eje de la estrategia de competitividad global de la ciudad (Peck, 2005).

En términos generales, las políticas culturales se fundamentan en la movilización de recursos para la promoción del mercado, donde la cultura se concibe como sistema de producción y factor de crecimiento económico. A su vez, la intervención gubernamental conlleva una lógica particular: el desa- 
rrollo de las industrias culturales y creativas, así como el de los denominados sectores culturales, se considera requisito primario para asegurar la calidad y el acceso a la cultura.

Un segundo tipo de respuesta tiene que ver con la cuestión de la cohesión social y, en ocasiones, con el elemento de proximidad. En este contexto, el factor proximidad se transforma en argumento central en la lógica de justificación de la acción pública en el ámbito de la cultura. El núcleo de las políticas de proximidad es, en buena medida, la promoción del acceso a la cultura, de las capacidades de expresión de las personas y el impulso del sentido de pertenencia colectiva. Ahora bien, más que la capacidad de las políticas para ofrecer respuestas al mismo tiempo diversificadas e integrales, democráticas y eficaces, la proximidad puede ser desplegada, en determinados casos, como factor de políticas «reparadoras». Antes que una aproximación integral y territorializada, las políticas de proximidad pueden resultar una respuesta conservadora al cuestionamiento de la representación política, en general, y al déficit de legitimidad de las políticas culturales, en particular (Barbieri et al., 2012).

Por un lado, la regeneración del discurso de la función social de la acción cultural es resultado de la caducidad de las instituciones y los principios de acción del modelo de las políticas de acceso. Los profesionales del sector cultural reclaman como propio un nuevo tipo de facultad: el conocimiento del territorio vinculado a la aparente capacidad de satisfacer los criterios de calidad cultural (Dubois and Laborier, 2003). Por otro lado, el discurso político sobre el impacto social de la cultura se centra en la capacidad de las políticas culturales para contribuir a la consecución de los objetivos de otras políticas públicas y sus agendas: educación, salud, medio ambiente, seguridad o urbanismo (Belfiore, 2006). Así, la perspectiva adoptada ha enfocado la atención en las externalidades de la cultura. Se defiende y argumenta en favor de la función instrumental de la acción cultural que recibe apoyo por parte de la administración pública (Subirats et al., 2008).

En definitiva, tanto las respuestas construidas sobre aspectos de desarrollo económico como de cohesión social se fundamentan en posiciones reactivas por parte de los gobiernos, como opciones de políticas culturales que podríamos llamar «defensivas». Ahora bien, reconociendo el impacto que tiene esta 
perspectiva para visibilizar la acción de las políticas culturales, es importante reconocer algunas de sus limitaciones más importantes.

Por un lado, resulta evidente el peligro que corremos en sobrevalorar los efectos de las políticas culturales. Sobrevalorar el impacto de las políticas culturales e incluso perder la mirada crítica sobre sus efectos. Además, podemos acabar generando expectativas y presiones desmesuradas sobre los agentes culturales. En muchos casos, en lugar de debatir sobre qué y cómo hacen su tarea en relación con problemáticas, diferencias y desigualdades tanto globales como locales, las organizaciones e instituciones culturales dedican su esfuerzo a demostrar si han contribuido a resolver objetivos de otras políticas sectoriales. Finalmente, esta tendencia nos ha alejado de la discusión sobre la capacidad de incidencia real de las políticas culturales en nuestra sociedad, lo que en definitiva resulta contraproducente para la legitimidad de la intervención pública en el ámbito de la cultura (Barbieri et al., 2011).

Estos aspectos quedan evidenciados cuando analizamos cómo las políticas culturales se han construido sobre la idea de la cultura como sustantivo, como objeto, incluso como esencia de un grupo o colectivo. Las políticas culturales se han estructurado mucho más en torno a «la» cultura que a «lo» cultural, a aquello que es cultural. Desarrollamos políticas de la cultura sustantiva y no de la cultura adjetiva. Poderes públicos y el sector cultural en su conjunto nos hemos refugiado en las políticas culturales como políticas de la cultura. Nos alejamos así de las políticas de lo cultural.

Un ejemplo en este sentido, que concierne a la actividad de quienes se dedican diariamente a la gestión de la cultura, es la información que se genera sobre los resultados de las políticas culturales. Hemos desarrollado sistemas de información cultural, como por ejemplo las cuentas satélite, que suponen un avance significativo. Pero la información que se genera sobre los resultados de las políticas culturales está enfocada mayoritariamente en la cuantificación de las actividades y productos del sector cultural, así como en el consumo cultural. Pensamos las políticas culturales como políticas de los sectores culturales (cine, teatro, etc.); pensamos los problemas culturales como problemas de los agentes del sector cultural, y pensamos los resultados de las políticas culturales en términos de consumo casi binario. 
En definitiva, generamos información sobre la cultura como sustantivo y mucho menos sobre la cultura como adjetivo. Conocemos el número de personas que trabajan en el sector cultural o, por ejemplo en algunos casos, el número de bibliotecas públicas por habitantes de una ciudad. Pero poco sabemos sobre quiénes entran en esas bibliotecas, cómo y con quién lo hacen, y cuáles son los efectos de su participación no sólo en términos individuales sino también colectivos. En definitiva, muy poco se ha avanzado en la construcción de herramientas que informen sobre las repercusiones que en términos de procesos sociales y de efectos transversales pueden tener las políticas culturales. Muy poco se ha avanzado en conocer (y sobre todo, en utilizar en la gestión cotidiana) información sobre los elementos afectivos, intangibles, de la experiencia y la práctica cultural, así como su vinculación con valores como la equidad o la justicia (Holden, 2004). Muy poco sabemos sobre la contribución (o no) de las políticas culturales en cuestiones como el desarrollo de identidades colectivas flexibles, la regeneración de vínculos sociales, el desarrollo personal autónomo y creativo, la democratización en la generación y el acceso al conocimiento, la revalorización de determinados colectivos (infancia, personas mayores) o la gobernanza inclusiva del territorio.

La cuestión del tipo de información y conocimiento que generamos no es un ejemplo menor. Y no lo es porque implica que desconocemos aquello que necesitamos: información y conocimiento relevante, entre otras cosas, para informar las decisiones sobre las políticas y la gestión cultural.

\section{Las políticas culturales en el interregno}

Los problemas a los que las políticas públicas se enfrentan hoy (y que por lo tanto no pueden evitar gestionar) resultan cada vez más complejos, inciertos y con más riesgos incorporados. Se trata de problemas condicionados por el desarrollo tecnológico y la emergencia de la sociedad informacional (Castells, 2000), por un desarrollo económico a escala global con un carácter financiero dominante y donde los símbolos y los signos adquieren un lugar central (Rifkin, 2000), y por el desarrollo de sociedades más heterogéneas e individualizadas (Bauman, 2003). Más recientemente, los cambios en el 
tipo de participación y producción cultural derivados de la consolidación de la diversidad cultural así como de la digitalización de la cultura (Ariño, 2010) desbordan la lógica de los equipamientos culturales tradicionales. A todo ello cabe sumar la reproducción de los cuestionamientos al rol de intermediación política tradicional de las instituciones públicas, así como la retracción de la inversión pública (Barbieri, 2012).

En definitiva, no sólo estamos experimentando cambios. Vivimos, en palabras de Zygmunt Bauman (2012), en un interregno, en un momento de cambio de época (Subirats, 2011). Éste es el escenario en el que se debaten las políticas culturales de hoy en día. Tanto los principios que sostenían el modelo de políticas de acceso como muchas de sus respuestas defensivas resultan hoy caducos, faltos de legitimidad. Mucho más si se trata de políticas que no cuentan con mecanismos para conocer la realidad en la que intervienen. Frente a ello, se vuelve cada vez más evidente un reto que no es nuevo pero sí de actualidad: asumir, entender la cultura no sólo como sustantivo sino también (o más bien) como adjetivo. Y construir entonces no ya políticas de la cultura sino políticas de lo cultural.

¿Qué quiere decir asumir el reto de impulsar políticas de lo cultural? Del pensamiento de investigadores como Appadurai (1996), García Canclini (2004), Barbero (2008) o Grimson (2003) hemos aprendido que la cultura no es sólo sustantiva y que es importante entenderla como adjetivo (la cultura como lo cultural). Lo cultural sería aquello que nos permite ser agentes, aquello que nos hace ser protagonistas en nuestras prácticas sociales. Lo cultural son las maneras en que como actores nos enfrentamos y negociamos, y por lo tanto, también cómo imaginamos aquello que compartimos. Confrontar y compartir forman parte indivisible de cualquier proceso cultural.

Esta mirada es una mirada política de la cultura. Hace hincapié en el carácter político de la cultura. Y por político no deberíamos entender simplemente juego de intereses partidarios o lucha para conseguir determinados recursos. Del pensamiento de los autores mencionados hemos aprendido que pensar en el sentido de la cultura como proceso político quiere decir pensarla como proceso de confrontaciones que se dan justamente porque participamos en contextos comunes y porque imaginamos aquello que compartimos. 
¿Es posible entonces desarrollar políticas culturales que recojan esta mirada de la cultura? ¿Es posible concebir políticas culturales que construyan lo público como algo heterogéneo, que no reduzcan lo que es público a lo institucional o estatal, y que trabajen con una idea de lo público como espacio de lo común (un espacio diverso y, por lo tanto, no exento de conflictos)? No tenemos certezas al respecto, pero sí que podemos afirmar la conexión entre este sentido de la cultura y un proceso de recorrido histórico y de renovada actualidad, que analizamos a continuación.

\section{De las políticas de acceso a las políticas de los bienes comunes}

La posibilidad de desarrollar políticas de lo cultural se plantea en este trabajo como modelo complementario (pero no sustitutivo) de las políticas de promoción del acceso a la oferta cultural. La necesidad de recuperar y enfatizar el sentido político de la cultura es un proceso que implica reequilibrios en el llamado sector cultural. Porque el sentido político de la cultura conecta con una mirada que entiende la cultura como parte de un proceso emergente en la actualidad: el de la reflexión y la acción en el ámbito de los bienes comunes.

Lo común, los commons, el procomún, los bienes comunes e incluso comunalizar, son palabras que se repiten en múltiples foros desde hace un cierto tiempo. Estas palabras emergen no sólo como conceptos analíticos sino también como poderosas herramientas de acción política, social y legal (Holder y Flessas, 2008), impulsadas por numerosos movimientos sociales de todo tipo y en diferentes espacios (ambiental, económico, urbano, digital, etc.).

Los bienes comunes y su estudio no son una nueva realidad, mucho menos en América Latina o incluso en Estados Unidos. La premio Nobel Elinor Ostrom analizó ampliamente hace más de dos décadas mecanismos de gestión de bienes comunes naturales (Ostrom, 1990). Más recientemente, el profesor de la Universidad de Harvard Yochai Benkler (2006) hizo lo propio con el mundo digital, estudiando iniciativas colaborativas como el software libre. Sin embargo, en el ámbito cultural todavía son incipientes los estudios al respecto. Todavía nos falta una perspectiva propia (aunque no aislada), que resulta necesaria si tenemos en cuenta las diferencias con los bienes de tipo natural y digital. 
Pero, ¿qué son exactamente los bienes comunes? En primer lugar, debemos entender que los bienes comunes no son ni espacios ni objetos. Los bienes comunes están compuestos por tres elementos: unos recursos, unas comunidades que comparten dichos recursos y unas normas desarrolladas por dichas comunidades con el objeto de hacer sostenible todo el proceso. Es decir, los bienes comunes son: $a$ ) sistemas de gobernanza o de gestión compartida de recursos, $b$ ) sistemas desarrollados por determinadas comunidades, y c) sistemas que tienen normas, reglas identificables. Esto quiere decir que hemos aprendido a mirar los bienes comunes menos como sustantivos (el bien común) y más como adjetivos (lo común). Lo que proponemos es adoptar una mirada similar también con la cultura y las políticas culturales.

En esta mirada, una de las primeras puntualizaciones que debemos tener en cuenta es el matiz del plural en la idea de bienes comunes. La cultura como parte de los bienes comunes y no tanto como bien común, porque no postulamos una posible superioridad moral de aquello que es cultural. La cultura no es una esfera elevada y separada de la sociedad o de la política. Y entonces, ¿por qué hablamos de la cultura como parte de los bienes comunes? Porque podemos plantear como hipótesis la necesidad de que las políticas culturales reconozcan este sentido de la cultura. Que las políticas culturales sean políticas de los bienes comunes.

Hablar de políticas públicas y bienes comunes, de la relación entre gobiernos y bienes comunes, tiene implicaciones significativas. El papel del Estado en relación con los bienes comunes ha sido ciertamente descrito como nocivo, incluso como depredador de muchos de los recursos y comunidades (Anthony y Campbell, 2011). Ahora bien, todavía restan infraanalizados los posibles roles alternativos que podrían desempeñar las políticas públicas (los gobiernos y sus actores) en los bienes comunes y, en este caso, en los bienes comunes culturales.

Una mirada de las políticas culturales como políticas de los bienes comunes, como políticas de lo común, nos obliga a dos cosas. Por un lado, si pensamos en la cultura como parte de los bienes comunes, debemos asumir que hablamos no sólo de recursos, ya sean intangibles - lenguas, expresiones diversas - o tangibles - equipamientos culturales, etc. - . Hablamos fundamentalmente de maneras colectivas de gestionar estos recursos. Es decir, las 
políticas culturales no deberían pensar la cultura solamente como objetos o servicios culturales, sino también poder identificar y reconocer las comunidades que gestionan de forma compartida estos recursos.

Por otro lado, si pensamos en la cultura como parte de los bienes comunes debemos reconocer el tercer elemento clave que comporta esta mirada. Además de los recursos y las comunidades, los bienes comunes incorporan normas propias, maneras de hacer. Si pensamos en las políticas culturales como políticas de los bienes comunes, deberíamos aceptar que la gestión colectiva de lo común comporta normas o reglas que son desarrolladas por las propias comunidades y que permiten su sostenibilidad.

En lugares donde las administraciones públicas están fuertemente jerarquizadas, pensar las políticas culturales como políticas de lo cultural, como políticas de los bienes comunes, supone asumir riesgos. Implica el reto de avanzar más allá de los principios que fundamentaban el modelo de políticas de acceso a la cultura. Podemos postular entonces las características y diferencias entre los modelos de las políticas de acceso y de las políticas de los bienes comunes. Tanto en la dimensión simbólica-sustantiva de las políticas como en la procesual-operativa (Gomà y Subirats, 1998).

\begin{tabular}{|c|c|c|c|}
\hline & & Políticas de acceso & Políticas de los bienes comunes \\
\hline \multirow{3}{*}{ 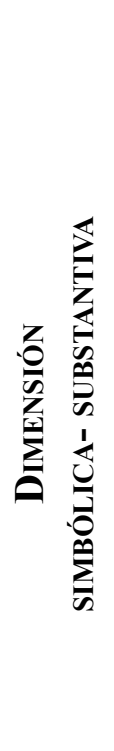 } & $\begin{array}{c}\text { Idea de la } \\
\text { cultura }\end{array}$ & $\begin{array}{l}\text { - Productos, bienes y } \\
\text { servicios públicos/pri- } \\
\text { vados. } \\
\text { - Sistema productivo: } \\
\text { creación, producción, } \\
\text { distribución, consumo. }\end{array}$ & $\begin{array}{l}\text { - Bienes comunes: recursos, nor- } \\
\text { mas, comunidades. } \\
\text { - Sistema híbrido sin delimitación } \\
\text { clara de roles. }\end{array}$ \\
\hline & $\begin{array}{l}\text { Lógica de } \\
\text { justifica- } \\
\text { ción de } \\
\text { la política } \\
\text { pública }\end{array}$ & $\begin{array}{l}\text { - Distributiva: equili- } \\
\text { brio del mercado. } \\
\text { - Creación de sistema } \\
\text { de identificación y pro- } \\
\text { moción económica. }\end{array}$ & $\begin{array}{l}\text { - Redistributiva: preservación y } \\
\text { fomento de bienes comunes. } \\
\text { - Retorno social: nuevas centralida- } \\
\text { des económicas, innovación social. }\end{array}$ \\
\hline & $\begin{array}{l}\text { Agenda } \\
\text { principal }\end{array}$ & $\begin{array}{l}\text { - Oferta: difusión (equi- } \\
\text { pamientos) y produc- } \\
\text { ción (industrias). } \\
\text { - Sectorial. }\end{array}$ & $\begin{array}{l}\text { - Demanda: formación (usuarios), } \\
\text { soporte (comunidades). } \\
\text {-I nterdisciplinar (tecnología, artes, } \\
\text { educación, etc.). }\end{array}$ \\
\hline
\end{tabular}




\begin{tabular}{|c|c|c|c|}
\hline \multirow{4}{*}{ 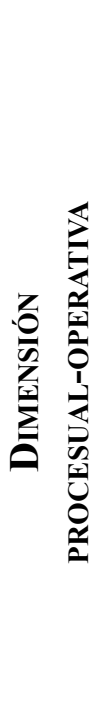 } & $\begin{array}{l}\text { Capacidad } \\
\text { de agencia } \\
\text { de las polí- } \\
\text { ticas }\end{array}$ & $\begin{array}{l}\text {-Sistema binario: } \\
\text { estatal-mercantil, go- } \\
\text { bierno-mercado, públi- } \\
\text { co-privado. }\end{array}$ & $\begin{array}{l}\text {-Sistema híbrido: inclusión de co- } \\
\text { munidades, policentrismo. }\end{array}$ \\
\hline & $\begin{array}{l}\text { Rol en la } \\
\text { creación de } \\
\text { valor }\end{array}$ & $\begin{array}{l}\text {-Fomentar la competiti- } \\
\text { vidad: interacción raci- } \\
\text { onal instrumental. }\end{array}$ & $\begin{array}{l}\text {-Promover la colaboración: respon- } \\
\text { sabilidad colaborativa. }\end{array}$ \\
\hline & $\begin{array}{l}\text { Tipo de } \\
\text { liderazgo }\end{array}$ & $\begin{array}{l}\text {-Administrativo: con- } \\
\text { trol vertical, departa- } \\
\text { mentalización horizon- } \\
\text { tal según subsectores. }\end{array}$ & $\begin{array}{l}\text {-Político: reconocimiento de la } \\
\text { interdependencia, garantía, trans- } \\
\text { parencia. }\end{array}$ \\
\hline & $\begin{array}{l}\text { Instrumen- } \\
\text { tos de rela- } \\
\text { ción }\end{array}$ & $\begin{array}{l}\text {-Subvención, mecenaz- } \\
\text { go, contratación directa. }\end{array}$ & $\begin{array}{l}\text {-Fondos de financiación colectiva, } \\
\text { red distribuida, fideicomiso, capital } \\
\text { riego. }\end{array}$ \\
\hline
\end{tabular}

Tabla 1. Elementos principales de las políticas de acceso y las políticas de los bienes comunes [Fuente: elaboración propia]

Sin repetir lo que la propia tabla presenta, cabe desarrollar dos grandes ámbitos de diferenciación entre el modelo de políticas de acceso y el de políticas de los bienes comunes. Una primera gran diferencia, que hemos apuntado previamente, se refiere a la idea de cultura que encontramos detrás de cada una de estas aproximaciones. En el modelo de las políticas de acceso, la cultura se entiende como producto o servicio. La cultura hace referencia a un sistema productivo donde encontramos claramente diferenciados creadores, productores, distribuidores y consumidores. Por su parte, en el modelo de políticas de lo común, la idea de cultura es la de bienes comunes culturales, es decir, complejos sistemas de gobernanza con comunidades que han desarrollado sus propias reglas para gestionar los recursos culturales. En este caso, no tenemos por qué encontrar claramente diferenciados los roles tradicionales de un sistema productivo, sino más bien todo lo contrario.

Pero las diferencias no se limitan a la dimensión conceptual o simbólica de las políticas culturales. También hablamos de diferencias en las formas de 
gobernar, en la dimensión operativa de las políticas. En concreto, en el rol del gobierno y en el tipo de liderazgo que puede ejercer. En el modelo de políticas de acceso, encontramos un gobierno que ejerce un rol que calificamos de administrativo, un liderazgo vertical de mando y control y una departamentalización horizontal de las políticas culturales según los subsectores (cine, teatro, etc.). La tarea que asume el gobierno es básicamente la de promover la competitividad, una interacción de tipo racional instrumental.

En cambio, en las políticas culturales que definimos como políticas de los bienes comunes, el rol de gobierno es entendido y asumido como plenamente político (en el más amplio y complejo sentido de la palabra). Se reconoce la interdependencia entre los actores y comunidades y se desarrollan instrumentos para promover la colaboración, la corresponsabilidad colaborativa. En definitiva, estaríamos ante gobiernos que (sin dejar de asumir su parte de responsabilidad) ofrecen su apoyo a modelos de gestión híbridos, que escapan del exceso de intermediación tradicional pero también de la mercantilización de las políticas.

\section{Políticas culturales, políticas de lo común: ¿qué rol para los gobiernos?}

En este marco que planteamos, resulta caduca la idea de interés público como algo determinado por una autoridad centralizada y abstracta. En un escenario de cambio de época, los poderes locales, la sociedad civil y muchas comunidades, tienen su propia concepción de lo común y de lo público; y han conseguido gestionar bienes comunes (naturales, culturales) de manera sostenible y socialmente justa.

¿Y cuáles son entonces las políticas culturales de lo común? Todavía no las conocemos en detalle, porque todavía son incipientes. Pero cabe pensar en aquellas políticas que más han buscado transformarse y reubicarse en el interregno en el que vivimos. Pensemos, por ejemplo, en las bibliotecas, museos y centros culturales que han dejado atrás su función como repositorios de cultura y exploran iniciativas colectivas de aprendizaje compartido y de difusión abierta de conocimiento. Instituciones culturales que se construyen ellas mismas como bienes comunes. 
Se trata de instituciones donde continúa existiendo un recurso central, como por ejemplo los libros o la información en el caso de las bibliotecas. También, sin duda, el gobierno mantiene un lugar importante en la comunidad que gestiona dicha institución; pero, a diferencia de lo que sucede en el modelo de políticas de acceso, ese rol de intermediación es significativamente distinto. Así, las reglas y las responsabilidades de cada uno de los agentes implicados en la sostenibilidad de la institución son compartidas y definidas de forma interdependiente.

Este proceso supone un nuevo reto para la legitimidad de la intervención gubernamental. Sobre todo para el tipo de intermediación que tradicionalmente han asumido las políticas públicas. Por eso, y conviene advertir de ello, un tipo de respuestas de los poderes públicos frente a este escenario puede no ser el que estamos planteando. En cambio, los gobiernos podrían optar por la desresponsabilización. El discurso de los bienes comunes funcionaría, en este caso, como nueva respuesta de carácter «defensivo» y con efecto relegitimador frente a las dificultades de financiación de las instituciones culturales públicas. Los bienes comunes funcionarían como respuesta conservadora que permitiría a los gobiernos salir del paso frente a la crisis de la representación política. Los bienes comunes serían el nuevo recurso de un conocido realismo mágico de la política.

De hecho, comienzan a desarrollarse las primeras acciones en este sentido, respuestas en el camino de la desresponsabilización de los gobiernos en la gestión de los bienes comunes culturales. En el ámbito específicamente cultural, uno de los ejes centrales a tener en cuenta para analizar el desarrollo de los bienes comunes y del papel de la política pública es la cuestión de la financiación. El tema de la financiación colectiva (o del crowdfunding, aunque este término pueda reducir la complejidad de estas nuevas prácticas) es un caso clave para el futuro de los bienes comunes culturales. Y también para el papel que van a asumir las políticas públicas al respecto.

Resulta sugerente observar cómo las primeras iniciativas gubernamentales en relación con el tema de la financiación colectiva están interpretando este fenómeno más bien como respuesta puntual a las dificultades económicas que muchas de las instituciones públicas han enfrentado en el marco del modelo de políticas de acceso. Un ejemplo es la campaña de financiación abierta a 
la ciudadanía que organizó recientemente el Museo de Bellas Artes de Lyon. Con el objetivo de poder adquirir la pintura de Jean-Auguste-Dominique Ingres, L'Aretin et l'envoye de Charles Quint, el museo impulsó una campaña de crowdfunding en búsqueda de micromecenas. A través de esta iniciativa, el museo consiguió recaudar ochenta mil euros, lo que supuso más del diez por ciento del total del coste de la obra. Más allá del caso concreto, el interés reside en analizar y debatir la posición de los gobiernos frente al fenómeno de la financiación colectiva. Actualmente, no sólo instituciones culturales de reducidas dimensiones sino grandes organizaciones estatales, se asumen como receptores de las contribuciones económicas de las personas, como beneficiarios del micromecenazgo, como destinatarios sin mayores responsabilidades en el desarrollo del fenómeno de la financiación colectiva.

Ahora bien, no todas las instituciones asumen un rol similar. Existen casos en los que, impulsados por las propias plataformas de financiación colectiva, se establecen vínculos más acordes con la profunda transformación que podría suponer este fenómeno para el sector cultural. Por ejemplo, la plataforma Goteo experimenta desde hace un tiempo con fórmulas como el «capital riego» o las «bolsas de inversión social» (Goteo, 2013). En estas iniciativas, los gobiernos (por ejemplo, del País Vasco o de Extremadura) se comprometen a aportar una suma equivalente a la que destinen las personas a título individual. Es decir, por cada euro recaudado por la plataforma con las contribuciones individuales, el gobierno se compromete a aportar otro euro más (hasta un límite acordado).

Lógicamente, éste es también un ejemplo concreto que no permite extraer conclusiones definitivas sobre el rol de las políticas públicas en los nuevos regímenes de financiación colectiva de la cultura. Sin embargo, sí que enfatiza la necesidad de continuar investigando hasta qué punto las nuevas formas de financiación (como el crowdfunding) se han desarrollado, en algunos casos, como bienes comunes.

\section{Reflexiones finales}

Más que concluir recuperando los argumentos centrales de este artículo, resulta relevante contribuir al debate sobre la necesidad de una agen- 
da de investigación sobre políticas culturales y bienes comunes. En este sentido, reproducimos conscientemente el sesgo que apunta Barbero (2008) en su decisión de investigar no aquello que nos haga ser optimistas, sino aquello que nos dé esperanzas. Investigar dinámicas como las que presenta este artículo puede generar conocimiento relevante y permitir alimentar la implementación de sistemas de gestión considerando estos aprendizajes.

Es relevante conocer de qué manera plataformas y colectivos han podido generar sus propias comunidades y han podido establecer normas compartidas para hacer sostenibles los procesos culturales. También es importante preguntarse por la ambigüedad que puede comportar el discurso de los bienes comunes, tanto en las dinámicas de desresponsabilización por parte de los gobiernos, como en las de exclusión en el seno de las comunidades, con conflictos que pueden provocar la pérdida del carácter público de los bienes comunes. Es decir, necesitamos conocer mejor cómo se han desarrollado los bienes comunes culturales y preguntarnos por el rol de las políticas públicas en estos procesos.

Las políticas culturales se enfrentan al reto de comprender y favorecer el desarrollo de los bienes comunes, por ejemplo a través de un régimen legal que ayude a maximizar el retorno social de estas iniciativas, sin que ello impida el desarrollo de estándares de calidad y remuneración de la autoría. Pero también se enfrentan a la necesidad de redefinir (y no suprimir) sus responsabilidades, contribuyendo a hacer de lo común algo que no pierda su carácter público, y viceversa.

\section{Referencias}

Anthony, D. y J. Campbell (2011): «States, Social Capital and Cooperation: Looking Back on Governing the Commons», International Journal of the Commons, Vol. 5, n. 2, pp. 284-302.

Appadurai, A. (1996): Modernity at Large: Cultural Dimensions of Globalization, University of Minnesota Press, Minneapolis.

Ariño, A. (2010): Prácticas culturales en España, Ariel, Barcelona.

Barbero, J. (2008): «Políticas de la comunicación y la cultura: Claves de la investigación», Dinámicas interculturales СIDOB (11). 
Barbieri, N. (2012): «Why Does Cultural Policy Change? Policy Discourse and Policy Subsystem: A Case Study Of The Evolution Of Cultural Policy In Catalonia», International Journal of Cultural Policy, 18, 1: pp. 13-30.

Barbieri, N.; A. Partal y E. Merino (2011): «Nuevas políticas, nuevas miradas y metodologías de evaluación. ¿Cómo evaluar el retorno social de las políticas culturales?», Papers Revista de Sociologia, 96, 2: pp. 477-500.

Barbieri, N; X. Fina y J. Subirats (2012): «Culture and Urban Policy. Dynamics and Effects of Third Sector Organisations in Barcelona», Revue Métropoles, 11, pp. 2-23.

Bauman, Z. (2003): Modernidad líquida. Fondo de Cultura Económica, México.

- (2012): «Times of Interregnum», Ethics and Global Politics, Vol. 5, 1: pp. 49-56.

Belfiore, E. (2006): «The Social Impacts of the Arts - Myth or Reality?». En MirzA, M. (ed.): Culture Vultures, Policy Exchange, London.

Benkler, Y. (2006): The Wealth of Networks: How Social Production Transforms Markets and Freedom, Yale U. Press, New Haven.

Bonet, L. y E. NÉGRIER (2008): La fin des culturelles nationales? Les politiques culturelles à l'épreuve de la diversité, La Découverte, Paris.

Castells, M. (2000): The Rise of the network society, Blackwell, Oxford.

Cherbo, J. y M. Wyszomirski (2000): The Public Life of the Arts in Ameri$c a$. Rutgers University Press, New Brunswick.

Dubois, V. y P. Laborier (2003): "The "Social" in the Institutionalisation of Local Cultural Policies in France and Germany», International Journal of Cultural Policy, 9, 2: 195-206.

Florida, R. (2002): The Rise of the Creative Class, Basic Books, New York.

García Canclini, N. (2004): Diferentes, desiguales y desconectados, Gedisa, Barcelona.

Goteo (2013): «Capital riego», disponible en: <http://goteo.org/service/resources?lang=es $>[04 / 11 / 2013]$.

Grimson, A. (2003): Tram(p)as de la comunicación y la cultura, Buenos Aires, Universidad de La Plata.

Hall, P. (2004): «Creativity, Culture, Knowledge and the City», Built Environment, 30 (3): 256- 258 .

Holder, J. y T. Flessas (2008): «Emerging Commons», Social \& Legal Studies, 17: 299-311. 
GLAESER, E. (2005): «Review of Richard Florida's "The Rise of the Creative Class"», Science and Urban Economics, 35: 593-596.

Gomà, R. y J. Subirats (1998): Políticas públicas en España: contenidos, redes de actores y niveles de gobierno, Ariel, Barcelona.

Holden, J. (2004): Capturing Cultural Value, Demos, London.

Knudsen, B.; R. Florida; G. Gates y K. Stolarick (2007): «Urban Density, Creativity, and Innovation», Working Paper of the Creative Class Group, The Martin Prosperity Institute, University of Toronto, Toronto.

Ostrom, E. (1990): Governing the Commons: The Evolution of Institutions for Collective Action. Cambridge University Press, London.

Peck, J. (2005): «Struggling with the creative class», International Journal of Urban and Regional Research, 29, 4: pp. 740-770.

Pratt, A. (2008): «Creative cities: the cultural industries and the creative class», Geografiska Annaler, series B, Human Geography, 90 (2): pp. 107-117.

Rıus, J. (2012): «Política cultural e hibridación de las instituciones culturales. El caso de Barcelona», Revista Española de Ciencia Política, 27: pp. 85-105.

Rodríguez Morató, A. (2005): «La reinvención de la política cultural a escala local: el caso de Barcelona», Sociedade e Estado, 20, 2: pp. 351-376.

Subirats, J. y I. Blanco (2009): «¿Todo lo urbano es social y todo lo social es urbano?: dinámicas urbanas y dilemas de políticas públicas», Medio ambiente y urbanización, 70, 1, pp. 3-13.

Subirats, J.; X. Fina; N. Barbieri; A. Partal y Merino, E. (2009): El retorn social de les politiques culturals, Departament de Cultura i Mitjans de Comunicació, Barcelona.

SubIRATs, J. (2011): Otra sociedad, ¿otra política?, Icaria, Barcelona.

Urfalino, P. (1996): L'invention de la politique culturelle, Hachette, Paris.

Wu, C. (2007): Privatizar la cultura, Akal, Madrid. 
